\title{
Impact of Intravenous Drug Use on HIV/AIDS among Women Prisoners: A Mathematical Modelling Approach
}

\author{
C. P. Bhunu and S. Mushayabasa \\ Department of Mathematics, P.O. Box MP 167, Mount Pleasant, Harare, Zimbabwe \\ Correspondence should be addressed to C. P. Bhunu; cpbhunu@gmail.com
}

Received 16 August 2013; Accepted 13 November 2013

Academic Editors: F.-B. Guo and B. Vasiev

Copyright (c) 2013 C. P. Bhunu and S. Mushayabasa. This is an open access article distributed under the Creative Commons Attribution License, which permits unrestricted use, distribution, and reproduction in any medium, provided the original work is properly cited.

\begin{abstract}
Intravenous drug use and tattooing remain one of the major routes of HIV/AIDS transmission among prisoners. We formulate and analyze a deterministic model for the role of intravenous drug use in HIV/AIDS transmission among women prisoners. With the aid of the Centre Manifold theory, the endemic equilibrium is shown to be locally asymptotically stable when the corresponding reproduction number is greater than unity. Analysis of the reproduction number and numerical simulations suggest that an increase in intravenous drug use among women prisoners as they fail to cope with prison settings fuels the HIV/AIDS epidemic in women prisoners. Failure to control HIV/AIDS among female prisoners may be a time bomb to their communities upon their release. Thus, it may be best to consider free needle/syringe exchange and drug substitution treatment programmes in women prisons as well as considering open prison systems for less serious crimes.
\end{abstract}

\section{Introduction}

The imprisonment of large numbers of drug addicts has the potential to create environments within which social networks that enhance the transmission of infectious diseases form [1-6]. Prisons are high-risk settings for HIV transmission. However, HIV prevention and treatment are not adequately developed and implemented to respond to HIV in prisons [7]. There is evidence to show that health programmes for the particular needs of imprisoned drug users are not enough in USA and Canada [8, 9]. In Russia, a study of intravenous drug users demonstrated the critical role of prisons in the transmission of HIV through high levels of needle (syringes) sharing among the imprisoned [10].

In many jurisdictions, a larger proportion of women than men are in prison for drug related offences [11]. Drug use and HIV infection are more prevalent among women in prison than among imprisoned men [12]. Women in prisons are more susceptible to self-harm through unsafe tattooing and injecting drug use [13]. In the absence of sterile injecting equipment, women, like men, will inject with used needles or home-made syringes, Doll [14] Like men, women get tattooed in prison [14]. In the absence of proper precautions and access to safe equipment tattooing can be a high-risk activity for the transmission of HIV $[15,16]$.

Women constitute a very small proportion of the total prison population, globally accounting $5 \%$ of the total prison population [15]. Women prisoners present specific challenges for correctional authorities as most of the existing prison facilities for women inmates have all being developed for men, who have historically accounted the largest proportion of the prison population [15, 17-19]. Scholarly research has called attention to the presence of HIV-infected women in prison and their lack of care while under institutional supervision [20]. With increase in female inmate population in the USA, the prison system is left with a small but significant population who suffers as a result of both HIV infection upon entering the criminal justice system, and contracting the infection while under correctional custody [21, 22]. Quite recently [23], we employed a similar approach to assess the impact of educational campaigns on $\mathrm{HCV}$ control among women prisoners. However, the present study differs from the previous work [23] in that here we are dealing with HIV/AIDS whose stages of disease progression are 
different from those of HCV. This results in the two models studied having different forces of infection. Furthermore, the potential benefits of open prison systems are explored here. Some studies [24] did analyse a model to predict the effects of nondisclosure by HIV positive people on the spread of the virus. Although disclosure is not the subject of this study, HIV status disclosure plays a significant role in the control of the epidemic. This is probably the first model that attempts to look into transmission dynamics of HIV/AIDS among female prisoners.

The rest of this paper is organized as follows. In the next section, the model and its basic properties are presented. In Section 3, we determine stability analysis of the equilibria states. Numerical simultaions are presented in Section 4 and finally the last section concludes the paper.

\section{Model Formulation}

Consider a population consisting of the following subpopulations: susceptible female prisoners nondrug injectors $S_{n}$, HIV positive only female prisoners nondrug injectors $I_{n_{h}}$, AIDS cases among female prisoners nondrug injectors $A_{n_{b}}$, susceptible female prisoners who are drug injectors $S_{d}$, HIV positive only female prisoners who are drug injectors $I_{d_{h}}$, and AIDS cases among female prisoners who are drug injectors $A_{d_{h}}$; thus the total population is given by

$$
\begin{gathered}
N=N_{n}+N_{d}, \\
N_{n}=S_{n}+I_{n_{h}}+A_{n_{h}}, \\
N_{d}=S_{d}+I_{d_{h}}+A_{d_{h}},
\end{gathered}
$$

where $N_{n}$ and $N_{d}$ are the total population of female nondrug using prisoners and total population of drug injecting female prisoners, respectively. Female prisoners are recruited into the prisons through committing different crimes at a constant rate $\Lambda$. We assume that fractions $\pi_{0}, \pi_{1}, \pi_{2}$, and $\pi_{3}\left(\sum_{n=0}^{3} \pi_{n}=\right.$ 1) are recruited into $S_{n}, I_{n_{h}}, S_{d}$, and $I_{d_{h}}$, respectively. Here, we further assume that AIDS patients are too sick to commit a crime as such there is no recruitment into the AIDS classes. So the AIDS cases we have in prisons are a direct result of progression to the AIDS stage while in prison. Due to peer pressure nondrug injecting female prisoners in $S_{n}$ and $I_{n_{h}}$ acquire drug injecting habits at rate $\rho$ to move into $S_{d}$ and $I_{d_{h}}$ classes, respectively. We assume that AIDS patients $A_{n_{h}}$ are now too sick to acquire drug injecting habits. Susceptible intravenous drug using female prisoners acquire HIV through sharing contaminated drug injection equipment at a rate

$$
\lambda_{h}=\frac{\beta_{h}\left(I_{d_{h}}+\theta A_{d_{h}}\right)}{N},
$$

where $\beta_{h}$ is the product of the probability of getting HIV infection per syringe sharing event and the number of people one shares it with; $\theta \in(0,1)$ accounts for a reduction in the rate of HIV transmission due to a decrease in the number of female prisoners willing to share drug injecting equipment

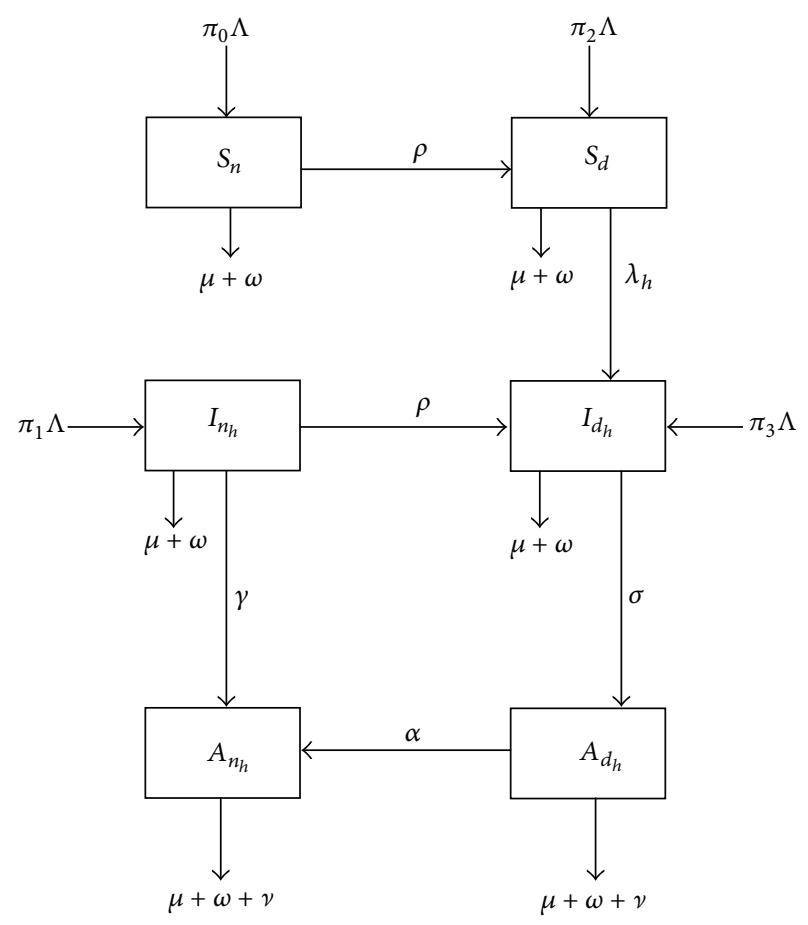

Figure 1: Model flow diagram.

with someone displaying AIDS symptoms. Natural mortality occurs in all classes at a rate $\mu$, while individuals in the AIDS stage suffer an additional disease-induced mortality at a rate $\nu$. Due to sickness drug injecting AIDS patients $A_{d_{h}}$ are assumed to leave drug injecting habits at a rate $\alpha$ and move into $A_{n_{h}}$. Upon completion of their sentences female prisoners leave prisons at rate $\omega$ back into their communities. The model flow diagram is depicted in Figure 1.

From the assumptions on the dynamics of the epidemic made above, the following are the model equations:

$$
\begin{gathered}
S_{n}^{\prime}=\Lambda \pi_{0}-(\rho+\omega+\mu) S_{n}, \\
I_{n_{h}}^{\prime}=\Lambda \pi_{1}-(\rho+\gamma+\omega+\mu) I_{n_{h}}, \\
A_{n_{h}}^{\prime}=\gamma I_{n_{h}}+\alpha A_{d_{h}}-(\omega+\mu+\nu) A_{n_{h}}, \\
S_{d}^{\prime}=\Lambda \pi_{2}+\rho S_{n}-\left(\lambda_{h}+\omega+\mu\right) S_{d}, \\
I_{d_{h}}^{\prime}=\Lambda \pi_{3}+\rho I_{n_{h}}+\lambda_{h} S_{d}-(\omega+\sigma+\mu) I_{d_{h}}, \\
A_{d_{h}}^{\prime}=\sigma I_{d_{h}}-(\omega+\alpha+\mu+\nu) A_{d_{h}} .
\end{gathered}
$$

The first octant of system (3) is positively invariant and attracting, and solutions starting in the first octant where all the variables are nonnegative stay there. Thus, system (3) will be analyzed in a suitable region $\mathscr{D} \subset \mathbb{R}_{+}^{6}$ given by

$$
\mathscr{D}=\left\{\left(S_{n}, I_{n_{h}}, A_{n_{h}}, S_{d}, I_{d_{h}}, A_{d_{h}}\right) \in \mathbb{R}_{+}^{6}: N \leq \frac{\Lambda}{\mu+\omega}\right\},
$$

which is positively invariant and attracting. Existence, uniqueness and continuation results for system (3) hold in this region. 


\section{Equilibrium States, Reproductive Number, and Stability}

Model system (3) has a disease-free equilibrium (DFE) which occurs when $\pi_{1}=\pi_{3}=0$ given by

$$
\begin{aligned}
\mathscr{V}^{0} & =\left(S_{n}^{0}, I_{n_{h}}^{0}, A_{n_{h}}^{0}, S_{d}^{0}, I_{d_{h}}^{0}, A_{d_{h}}^{0}\right) \\
& =\left(\frac{\Lambda \pi_{0}}{\rho+\omega+\mu}, 0,0, \frac{\Lambda\left(\rho+(\omega+\mu) \pi_{2}\right)}{(\omega+\mu)(\rho+\omega+\mu)}, 0,0\right) .
\end{aligned}
$$

Following Van Den Driessche and Watmough [25], we have

$$
\mathscr{R}_{0_{f}}=\frac{\beta_{h}\left(\rho+(\mu+\omega) \pi_{2}\right)}{(\mu+\sigma+\omega)(\mu+\omega+\rho)}\left(1+\frac{\theta \sigma}{\alpha+\mu+\nu+\omega}\right),
$$

which is defined as the number of secondary HIV infections produced by one infected female individual through the sharing of nonsterile syringes/needles in a prison. Theorem 1 follows from Van Den Driessche and Watmough [25].

Theorem 1. The disease-free equilibrium $\mathscr{V}^{0}$ is locally asymptotically stable when $\mathscr{R}_{0_{f}}<1$ and unstable otherwise.

Analysis Reproduction Number $\mathscr{R}_{0_{f}}$. Here we answer some issues pertaining to drug misuse in prisons by way of analysing the basic reproduction number $\mathscr{R}_{0_{f}}$ in an effort to understand the dynamics of HIV infections in female prisons. First, let us consider the effect intravenous drug injections have on HIV transmission. This is done by partially differenting $\mathscr{R}_{0_{f}}$ with respect to $\rho$ to obtain

$$
\begin{aligned}
\frac{\partial \mathscr{R}_{0_{f}}}{\partial \rho}= & \frac{\beta_{h}}{\mu+\sigma+\omega}\left(1+\frac{\theta \sigma}{\alpha+\mu+\nu+\omega}\right) \\
& \times \frac{\left(1-\pi_{2}\right)(\mu+\omega)}{(\mu+\omega+\rho)^{2}}>0 .
\end{aligned}
$$

The fact that (7) is positive suggests that an increase in females becoming intravenous drug users in prisons results in an increase of HIV related infections in women prisoners. Most women in prison resort to drug use and tattooing in prisons due to failure to cope with stressful prison conditions. Against this background it may be best to consider open prison systems for less serious crimes so that people may serve their sentences while coming from their homes. In this way they are surrounded by their families most of the time and hence are able to defeat the temptations to engage in intravenous drug use. Now consider

$$
\begin{aligned}
\lim _{\pi_{2} \rightarrow 0} \mathscr{R}_{0_{f}}=\mathscr{R}_{0_{f d}}= & \frac{\beta_{h} \rho}{(\mu+\sigma+\omega)(\mu+\omega+\rho)} \\
& \times\left(1+\frac{\sigma \theta}{\alpha+\mu+\nu+\omega}\right) .
\end{aligned}
$$

Substracting $\mathscr{R}_{0_{f d}}$ from $\mathscr{R}_{0_{f}}$ we have

$$
\begin{aligned}
\mathscr{R}_{0_{f}}-\mathscr{R}_{0_{f d}}= & \frac{\beta_{h} \pi_{2}(\mu+\omega)}{(\mu+\sigma+\omega)(\mu+\omega+\rho)} \\
& \times\left(1+\frac{\sigma \theta}{\alpha+\mu+\nu+\omega}\right)>0 .
\end{aligned}
$$

The issue of (9) being positive suggests that reducing the number of intravenous drug users in prisons reduces the spread of HIV in prisons. While theoretically this is feasible, in reality this is not feasible as drug users are more associated with crime than the general population. In light of that, perhaps, it is more feasible to have free needle exchange in prisons. This will assist in ensuring that female convicts do not share contaminated syringes. Furthermore, provision of drug substituting treatment will be of beneficial use to prisoners.

3.1. Endemic Equilibrium. In terms of the HIV and drug induction forces of infection $\lambda_{h}^{*}$ and $\rho$, the endemic equilibrium is given by $\mathscr{V}^{*}=\left(S_{n}^{*}, I_{n_{h}}^{*}, A_{n_{h}}^{*}, S_{d}^{*}, I_{d_{h}}^{*}, A_{d_{h}}^{*}\right)$, where

$$
S_{n}^{*}=\frac{\Lambda \pi_{0}}{\rho+\omega+\mu}, \quad I_{n_{h}}^{*}=\frac{\Lambda \pi_{0}}{\rho+\gamma+\omega+\mu},
$$

$$
\begin{aligned}
A_{n_{h}}^{*}= & \frac{\gamma \Lambda \pi_{0}}{(\omega+\mu+\nu)(\rho+\gamma+\omega+\mu)} \\
& +\frac{\alpha \sigma \Lambda}{(\omega+\mu+\nu)(\omega+\alpha+\mu+\nu)(\omega+\mu+\sigma)} \\
& \times\left(\frac{\left(\pi_{1}+\pi_{3}\right) \rho+\pi_{3}(\gamma+\omega+\mu)}{\rho+\gamma+\omega+\mu}\right. \\
& \left.+\frac{\lambda_{h}^{*}\left(\pi_{2}(\rho+\mu+\omega)+\pi_{0} \rho\right)}{\left(\lambda_{h}^{*}+\mu+\omega\right)(\rho+\mu+\omega)}\right), \\
I_{d}^{*}= & \frac{\Lambda\left(\pi_{2}(\rho+\mu+\omega)+\pi_{0} \rho\right)}{(\rho+\mu+\omega)\left(\lambda_{h}^{*}+\mu+\omega\right)}, \\
& +\frac{\Lambda\left(\lambda_{h}^{*}\left(\pi_{2}(\rho+\mu+\omega)+\pi_{0} \rho\right)\right.}{(\rho+\gamma+\omega+\mu)(\omega+\sigma+\mu)}, \\
A_{d_{h}}^{*}= & \frac{(\omega+\mu)(\rho+\mu+\omega)\left(\lambda_{h}^{*}+\mu+\omega\right)}{(\omega+\alpha+\mu+\nu)(\omega+\mu+\sigma)} \\
& \times\left(\pi_{1}+\pi_{3}\right) \rho+\pi_{3}(\gamma+\omega+\mu) \\
\rho+\gamma+\omega+\mu & \left(\lambda_{h}^{*}+\mu+\omega\right)(\rho+\mu+\omega)
\end{aligned} .
$$


To establish the local asymptotic stability of this equilibrium point, we use the Centre Manifold theory [26] as illustrated by Castillo-Chavez and Song [27]. To apply the Centre Manifold theory we make the following change of variables: $S_{n}=$ $x_{1}, I_{n_{h}}=x_{2}$, and $A_{n_{h}}=x_{3}, S_{d}=x_{4}, I_{d_{h}}=x_{5}, A_{n_{h}}=x_{6}$ so that $\sum_{n=1}^{6} x_{n}$. We now use the notation of the form $X=$ $\left(x_{1}, x_{2}, x_{3}, x_{4}, x_{5}, x_{6}\right)^{T}$. Then model system (3) can be written as $d X / d t=F=\left(f_{1}, f_{2}, f_{3}, f_{4}, f_{5}, f_{6}\right)^{T}$, where

$$
\begin{gathered}
x_{1}^{\prime}(t)=f_{1}=\Lambda \pi_{0}-(\rho+\omega+\mu) x_{1}, \\
x_{2}^{\prime}(t)=f_{2}=\Lambda \pi_{1}-(\rho+\omega+\gamma+\mu) x_{2},
\end{gathered}
$$

$$
\begin{gathered}
x_{3}^{\prime}(t)=f_{3}=\gamma x_{2}+\alpha x_{6}-(\mu+\omega+\nu) x_{3}, \\
x_{4}^{\prime}(t)=f_{4}=\Lambda \pi_{2}+\rho x_{1}-\frac{\beta_{h}\left(x_{5}+\theta x_{6}\right) x_{4}}{\sum_{n=1}^{6} x_{n}}-(\omega+\mu) x_{4}, \\
x_{5}^{\prime}(t)=f_{5}=\Lambda \pi_{3}+\rho x_{2} \\
+\frac{\beta_{h}\left(x_{5}+\theta x_{6}\right) x_{4}}{\sum_{n=1}^{6} x_{n}}-(\omega+\sigma+\mu) x_{5}, \\
x_{6}^{\prime}(t)=f_{6}=\sigma x_{5}-(\mu+\omega+\alpha+\nu) x_{6} .
\end{gathered}
$$

The Jacobian matrix of system (11) at $\mathscr{V}^{0}$ is given by

$$
\left.\begin{array}{cc}
0 & 0 \\
0 & 0 \\
0 & \alpha \\
-\frac{\beta_{h}\left(\rho+\pi_{2} A_{1}\right)}{A_{2}} & -\frac{\beta_{h} \theta\left(\beta_{d}+\pi_{2} A_{1}\right)}{A_{2}} \\
\frac{\beta_{h}\left(\rho+\pi_{2} A_{1}\right)}{A_{2}}-A_{4} & \frac{\beta_{h} \theta\left(\rho+\pi_{2} A_{1}\right)}{A_{2}} \\
\sigma & -\left(A_{3}+\alpha\right)
\end{array}\right],
$$

with $A_{1}=\omega+\mu, A_{2}=A_{1}+\rho, A_{3}=A_{1}+\nu$, and $A_{4}=A_{1}+\sigma$.

It can be shown from $J\left(\mathscr{V}^{0}\right)$ in (12), the reproduction number of system (11) is

$$
\mathscr{R}_{0_{f}}=\frac{\beta_{h}\left(\rho+(\mu+\omega) \pi_{2}\right)}{(\mu+\sigma+\omega)(\mu+\omega+\rho)}\left(1+\frac{\theta \sigma}{\alpha+\mu+\nu+\omega}\right) .
$$

If $\beta_{h}$ is taken as a bifurcation point and if we consider the case $\mathscr{R}_{0_{f}}=1$ and $\beta_{d_{h}}$ we have

$$
\beta_{h}=\beta_{h}^{*}=\frac{(\mu+\sigma+\omega)(\mu+\omega+\rho)(\alpha+\mu+\nu+\omega)}{(\alpha+\mu+\nu+\omega+\sigma \theta)\left(\rho+(\mu+\omega) \pi_{2}\right)} .
$$

Noting that the linearised system (11) with $\beta_{h}=\beta_{h}^{*}$ has simple zero eigenvalue, hence, the Centre Manifold theory [26] can be used to analyze the dynamics of system (11) near $\beta_{h}=\beta_{h}^{*}$. It can be shown that the Jacobian of system (11) at $\beta_{h}=\beta_{h}^{*}$ has a right eigenvector with the zero eigenvalue given by $u=$ $\left[u_{1}, u_{2}, u_{3}, u_{4}, u_{5}, u_{6}\right]^{T}$, where

$$
\begin{gathered}
u_{1}=u_{2}=0, \quad u_{3}=\frac{\alpha}{\omega+\mu+\nu} u_{6}, \quad u_{6}>0, \\
u_{5}=\frac{\mu+\alpha+\omega+v}{\sigma} u_{6}, \\
u_{4}=-\frac{\beta_{h}^{*}\left(\rho+(\omega+\mu) \pi_{2}\right)(\mu+\alpha+\omega+\nu+\theta \sigma)}{(\omega+\mu)(\omega+\mu+\rho) \sigma} u_{6} .
\end{gathered}
$$

The left eigenvector of $J\left(\mathscr{V}^{0}\right)$ is given by $v=\left[v_{1}, v_{2}, v_{3}\right.$, $\left.v_{4}, v_{5}, v_{6}\right]^{T}$, where

$$
\begin{gathered}
v_{1}=v_{3}=v_{4}=0, \\
v_{2}=\frac{\rho(\mu+\alpha+\omega+\nu)(\mu+\omega+\rho)}{\beta_{h}^{*} \theta\left(\pi_{2}(\mu+\omega)+\rho\right)(\omega+\mu+\gamma+\rho)} v_{6}, \\
v_{5}=\frac{(\mu+\alpha+\omega+\nu)(\mu+\omega+\rho)}{\beta_{h}^{*} \theta\left(\pi_{2}(\mu+\omega)+\rho\right)} v_{6}, \quad v_{6}>0 .
\end{gathered}
$$


In order to establish the existence of a bifurcation, we use Theorem 4 in [27].

Computation of $a$ and $b$. For model system (11), the associated nonzero derivatives of $F$ associated with $b$ at $\mathscr{V}^{0}$ as noted in Theorem 4 in [27] are given by

$$
\begin{gathered}
\frac{\partial^{2} f_{4}}{\partial \beta_{h}^{*} \partial x_{5}}=-\frac{\rho+(\omega+\mu) \pi_{2}}{\rho+\omega+\mu}, \\
\frac{\partial^{2} f_{4}}{\partial \beta_{h}^{*} \partial x_{6}}=-\frac{\theta\left(\rho+(\omega+\mu) \pi_{2}\right)}{\rho+\omega+\mu}, \\
\frac{\partial^{2} f_{5}}{\partial \beta_{h}^{*} \partial x_{5}}=\frac{\rho+(\omega+\mu) \pi_{2}}{\rho+\omega+\mu}, \\
\frac{\partial^{2} f_{5}}{\partial \beta_{h}^{*} \partial x_{6}}=-\frac{\theta\left(\rho+(\omega+\mu) \pi_{2}\right)}{\rho+\omega+\mu} .
\end{gathered}
$$

It follows from (15)-(17) that

$$
\begin{aligned}
b & =\sum_{k, i=1}^{6} v_{k} u_{i} \frac{\partial^{2} f_{k}}{\partial x_{i} \partial \beta_{h}^{*}}\left(\mathscr{V}^{0}\right) \\
& =\frac{(\mu+\alpha+\omega+\nu)(\mu+\alpha+\omega+\nu+\sigma \theta)}{\sigma \theta \beta_{h}^{*}} u_{6} v_{6}>0 .
\end{aligned}
$$

The non-zero partial derivatives of $F$ associated with $a$ at $\mathscr{V}^{0}$ as noted in Theorem 4 in [27] are given by

$$
\begin{gathered}
\frac{\partial^{2} f_{5}}{\partial x_{1} \partial x_{5}}=\frac{\partial^{2} f_{5}}{\partial x_{5} \partial x_{1}}=\frac{\partial^{2} f_{5}}{\partial x_{2} \partial x_{5}}=\frac{\partial^{2} f_{5}}{\partial x_{5} \partial x_{2}} \\
=-\frac{\beta_{h}^{*}(\omega+\mu)\left(\rho+(\omega+\mu) \pi_{2}\right)}{\Lambda(\rho+\omega+\mu)}, \\
\frac{\partial^{2} f_{5}}{\partial x_{1} \partial x_{6}}=\frac{\partial^{2} f_{5}}{\partial x_{6} \partial x_{1}}=-\frac{\beta_{h}^{*} \theta(\omega+\mu)\left(\rho+(\omega+\mu) \pi_{2}\right)}{\Lambda(\rho+\omega+\mu)}, \\
\frac{\partial^{2} f_{5}}{\partial x_{2} \partial x_{6}}=\frac{\partial^{2} f_{5}}{\partial x_{6} \partial x_{2}}=-\frac{\beta_{h}^{*} \theta(\omega+\mu)\left(\rho+(\omega+\mu) \pi_{2}\right)}{\Lambda(\rho+\omega+\mu)} \\
\frac{\partial^{2} f_{5}}{\partial x_{3} \partial x_{5}}=\frac{\partial^{2} f_{5}}{\partial x_{5} \partial x_{3}}=-\frac{\beta_{h}^{*}(\omega+\mu)\left(\rho+(\omega+\mu) \pi_{2}\right)}{\Lambda(\rho+\omega+\mu)} \\
\frac{\partial^{2} f_{5}}{\partial x_{3} \partial x_{6}}=\frac{\partial^{2} f_{5}}{\partial x_{6} \partial x_{3}}=-\frac{\beta_{h}^{*} \theta(\omega+\mu)\left(\rho+(\omega+\mu) \pi_{2}\right)}{\Lambda(\rho+\omega+\mu)} \\
\frac{\partial^{2} f_{5}}{\partial x_{4} \partial x_{5}}=\frac{\partial^{2} f_{5}}{\partial x_{5} \partial x_{4}}=\frac{\beta_{h}^{*} \pi_{0}(\omega+\mu)^{2}}{\Lambda(\rho+\omega+\mu)}, \\
\frac{\partial^{2} f_{5}}{\partial x_{4} \partial x_{6}}=\frac{\partial^{2} f_{5}}{\partial x_{6} \partial x_{4}}=\frac{\beta_{h}^{*} \theta \pi_{0}(\omega+\mu)^{2}}{\Lambda(\rho+\omega+\mu)}, \\
\frac{\partial x_{5}^{2}}{\Lambda}-\frac{2 \beta_{h}^{*}(\omega+\mu)\left(\rho+(\omega+\mu) \pi_{2}\right)}{\Lambda(\rho+\omega+\mu)}
\end{gathered}
$$

$$
\begin{gathered}
\frac{\partial^{2} f_{5}}{\partial x_{6}^{2}}=-\frac{2 \beta_{h}^{*} \theta(\omega+\mu)\left(\rho+(\omega+\mu) \pi_{2}\right)}{\Lambda(\rho+\omega+\mu)} \\
\frac{\partial^{2} f_{5}}{\partial x_{5} \partial x_{6}}=\frac{\partial^{2} f_{5}}{\partial x_{6} \partial x_{6}}=-\frac{\beta_{h}^{*}(1+\theta)(\omega+\mu)\left(\rho+(\omega+\mu) \pi_{2}\right)}{\Lambda(\rho+\omega+\mu)} .
\end{gathered}
$$

It follows from (15), (16), and (19) that

$$
\begin{aligned}
a= & \sum_{k, i, j=1}^{6} v_{k} u_{i} u_{j} \frac{\partial^{2} f_{k}}{\partial x_{i} \partial x_{j}}\left(\mathscr{V}^{0}\right) \\
= & -\frac{2(\omega+\mu)(\omega+\mu+\nu+\sigma)(\omega+\mu+\nu+\alpha)^{2}}{\sigma \Lambda(\omega+\mu+\nu)} \\
& \times\left(1+\frac{\omega+\mu+\nu+\alpha}{\sigma \theta}\right) u_{6}^{2} v_{6} \\
& -\frac{2(\omega+\mu+\alpha+\nu+\theta \sigma)^{2}(\omega+\mu+\alpha+\nu)(\mu+\omega) \beta_{h}^{*} \pi_{0}}{\theta \sigma^{2} \Lambda(\omega+\mu+\rho)} \\
& \times u_{6}^{2} v_{6}<0 .
\end{aligned}
$$

It is worth noting from (20) that $a$ is negative. Using Theorem 4 [27] item (iv) we establish the following result.

Theorem 2. The endemic equilibrium point $\mathscr{V}^{*}$ is locally asymptotically stable for $\mathscr{R}_{0_{f}}>1$ but close to 1 .

\section{Numerical Simulations}

In this section, we carry out detailed numerical simulations using Matlab programming language to assess the transmission dynamics of HIV in prisons housing female prisoners. The parameter values that we use for numerical simulations are in Table 1.

In Table 1, NPA denotes National Prison Administration (Zimbabwe); $a^{*}, b^{*}$, and $c^{*}$ denote parameter values adapted from Green and Al-Fwzan [28], Bhunu et al. [29], and Bhunu et al. [30], respectively. For influence of peer pressure forces influencing one to become an IDU, we used values adapted from Bhunu et al. [30], which are peer pressure forces necessary for one to start smoking, for the sake of illustration.

Figure 2 is a graphical representation showing the effects of intravenous drug use on HIV and AIDS cases in female prisons. Figures 2(a) and 2(b) show that total HIV-only and AIDS cases increase with increase in the percentage of intravenous drug users, respectively. This suggests that decreasing the levels of intravenous drug use will also result in a decrease in levels of HIV/AIDS prevalence in prisons. Theoretically, this is feasible but in the real world this is not possible to achieve. Currently, while the problem of intravenous drug use exists in female prisons, authorities have not yet acknowledged its existence. Perhaps the answer to the problem of intravenous drug use among female prisons is accepting that it exists and then offering free needle exchange and/or drug substitution treatment. 
TABLE 1: Model parameters and their interpretations.

\begin{tabular}{lccc}
\hline Parameter & Symbol & Value & Source \\
\hline Recruitment rate & $\Lambda$ & $0.00163 \mathrm{yr}^{-1} * 3 * 10^{6}$ & $0.02 \mathrm{yr}^{-1}$ \\
Natural mortality rate & $\mu$ & $0.1 \mathrm{yr}^{-1}$ & $\mathrm{NPA}^{*}$ \\
Natural rate of progression to AIDS & $\gamma, \sigma$ & 0.25 & $b^{*}$ \\
Modification parameter & $\theta$ & $0.4 \mathrm{yr}^{-1}$ & $0.562 \mathrm{yr}^{-1}$ \\
AIDS related death rate & $\beta_{h}$ & $0.4 \mathrm{yr}^{-1}$ & $b^{*}$ \\
Product of effective contact rate for HIV infection and & $\rho$ & $0.3 \mathrm{yr}^{-1}$ \\
probability of HIV transmission per drug injection & $\alpha$ & $0.25 \mathrm{yr}^{-1}$ \\
Rate of becoming a drug user & $\omega$ & $a^{*}$ \\
Rate of quitting drug misuse of sickness & $\pi_{0}^{*}, \pi_{1}, \pi_{2}, \pi_{3}$ & $c^{*}$ \\
Rate of release from prison & & $0.375,0.125,0.375,0.125$ \\
Proportion recruited into $S_{n}, I_{n_{h}}, S_{d}$, and $I_{d_{h}}$ classes & Assumed & Assumed \\
\hline
\end{tabular}

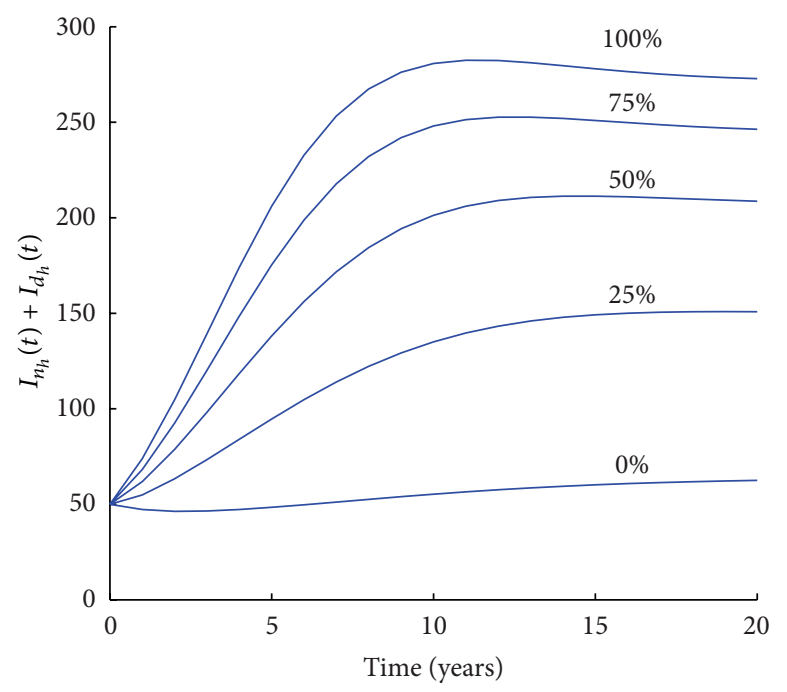

(a)

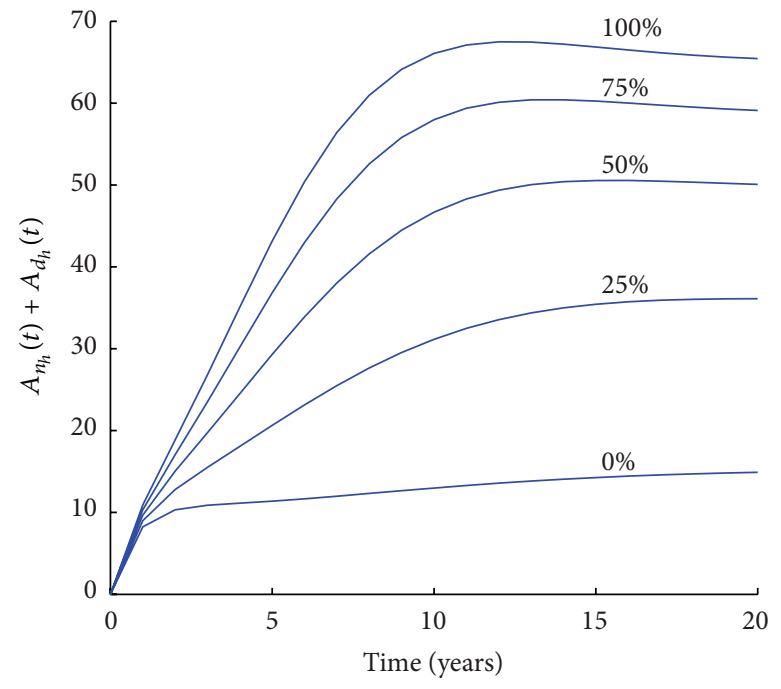

(b)

FIGURE 2: Simulations of model system (3) showing the effects of varying the percentage of intravenous drug users on the population of HIV positive people and AIDS cases in female prisoners. Parameter values used are in Table 1.

Figure 3 is a graphical representation showing how HIV and AIDS fare in 100\% and $0 \%$ of intravenous drug using female prisoner populations. In Figure 3 the HIV and AIDS population is depicted for various initial conditions. The HIV and AIDS cases converge to their distinct endemic equilibrium states depending on the level of intravenous drug use in the female prison population as shown in Figures $3(\mathrm{a})$ and 3(b), respectively. The higher the percentage of intravenous drug users, the larger the numbers of the HIVonly and AIDS cases at the endemic equilibrium point. This suggests that control of drug use in female prisons may provide an answer to the problem of HIV/AIDS among female prisoners.

\section{Discussion}

A mathematical model has been presented in attempt to understand the transmission dynamics of HIV/AIDS among female prisoners with particular reference to female prisoners in Zimbabwe. Drug use in female prisons is two way: (i) sharing of unsterile needles/syringes enhances the transmission of HIV and (ii) flashing blood that is drawing blood from someone who has injected herself with a drug and inject the blood into herself on its own exposes the injector to the HIV infection. Analysis of the reproduction number has shown that (i) a reduction in drug use results in a decrease of HIV/AIDS prevalence among female prisoners and (ii) release of prisoners may also act in reducing the concentration HIV/AIDS cases in prisons. The latter fact is not feasible, but perhaps implementing opening prison systems where prisoners of less serious crimes are allowed to serve their sentences while staying with their families enables female prisoners to cope with stressful prison conditions. Open prison systems will reduce the influence of peer pressure among prisoners as they will have moral and psychological support from the family which does not exist in enclosed female prison systems. Numerical simulations carried out also support the analytic results that increase 


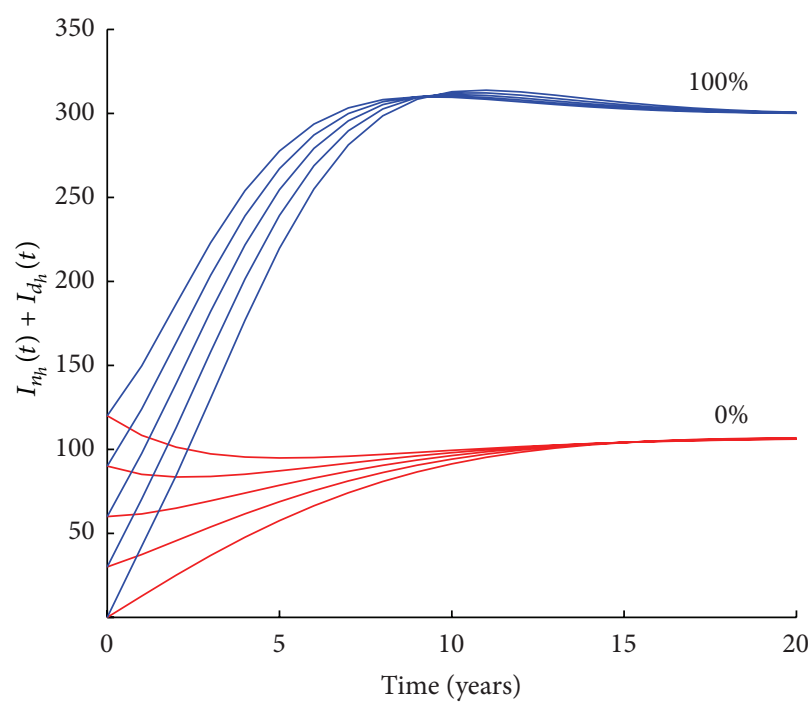

(a)

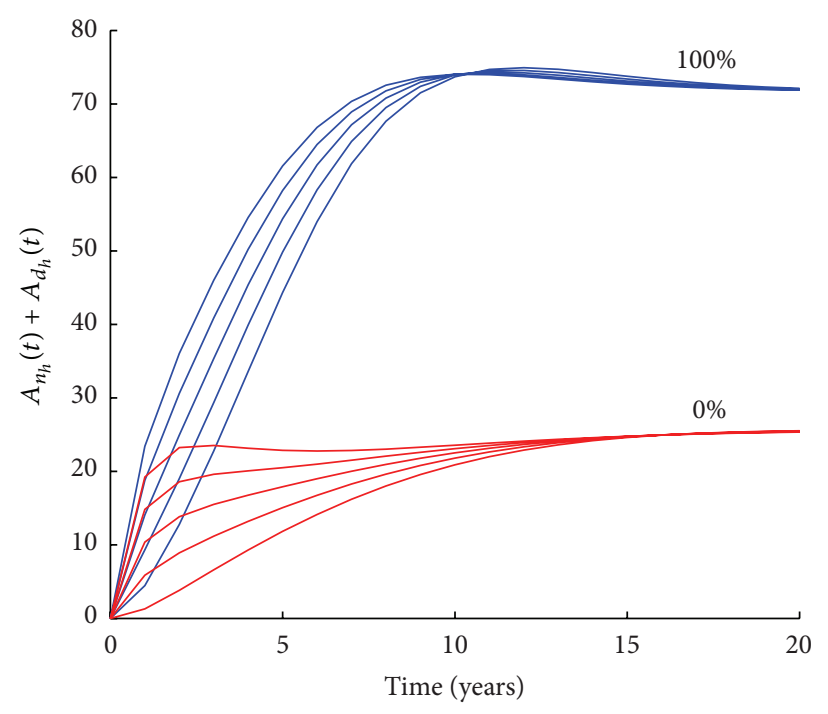

(b)

FIGURE 3: Time series evolution of the HIV-only infected individuals and those displaying symptoms of AIDS for various initial conditions when all female prisoners are made of nondrug users and when they are all intravenous users. Parameter values used are in Table 1.

in drug use and tattooing increases HIV/AIDS prevalence among women prisoners. The result of this study has a public health implication considering high rates of syringe lending and borrowing in prisons. This might explain why there are more HIV cases in prisons than the general population in the case of the USA $[2,3]$ and this might be the case world wide. HIV-infected women in prison pose a risk to their communities upon release from prison; so in the absence of open prison systems, it may be best to have mandatory HIV/AIDS screening and gender-specific educational programmes. This will reduce the prevalence of high-risk behaviours and lower HIV transmission in female prisons, thus reducing postrelease public health threat. Given the high levels of HIV in prisons, about three and half times higher among prisoners than the general population, it may be best to consider the introduction of needle/syringe free exchange programme and drug substitution treatment ways of keeping in check with HIV transmission in women prisons. The study is presented here is not exhaustive; it can be extended to include the contribution of male prison staff into the dynamics of HIV transmission among female prisoners as the male staff are the most likely people who sexually abuse female prisoners while incarcerated.

\section{References}

[1] A. Buavirat, K. Page-Shafer, G. J. P. van Griensven et al., "Risk of prevalent HIV infection associated with incarceration among injecting drug users in Bangkok, Thailand: case-control study," British Medical Journal, vol. 326, no. 7384, pp. 308-310, 2003.

[2] Centers for Disease Control and Prevention CDC, "HIV transmission among male inmates in a state prison system-Georgia, 1992-2005," Morbidity and Mortality Weekly Report, vol. 55, no. 15, pp. 421-426, 2006.
[3] Centers for Disease Control and Prevention CDC and C. D. C. Prevention, "National Center for HIV/AIDS, Viral Hepatitis, STD, and TB Prevention. Prevention and control of tuberculosis in correctional and detention facilities: recommendations from CDC, endorsed by the Advisory Council for the Elimination of Tuberculosis, the National Commission on Correctional Health Care, and the American Correctional Association," Morbidity and Mortality Weekly Report, vol. 55, no. 9, p. 144, 2006.

[4] Centers for Disease Control and Prevention, "Tuberculosis outbreaks in prison housing units for HIV-infected inmatesCalifornia, 1995-1996," Morbidity and Mortality Weekly Report, vol. 48, no. 4, p. 7982, 1999.

[5] N. Crofts, T. Stewart, P. Hearne, X. Y. Ping, A. M. Breschkin, and S. A. Locarnini, "Spread of bloodborne viruses among Australian prison entrants," British Medical Journal, vol. 310, no. 6975, pp. 285-288, 1995.

[6] K. A. Dolan and A. Wodak, "HIV transmission in a prison system in an Australian State," Medical Journal of Australia, vol. 171, no. 1, pp. 14-17, 1999.

[7] K. Dolan, B. Kite, E. Black, C. Aceijas, and G. V. Stimson, "HIV in prison in low-income and middle-income countries," Lancet Infectious Diseases, vol. 7, no. 1, pp. 32-41, 2007.

[8] T. P. Flanigan, J. D. Rich, and A. Spaulding, "HIV care among incarcerated persons: a missed opportunity," AIDS, vol. 13, no. 17, pp. 2475-2476, 1999.

[9] R. E. Martin, F. Gold, W. Murphy, V. Remple, J. Berkowitz, and D. Money, "Drug use and risk of bloodborne infections. A survey of female prisoners in British Columbia," Canadian Journal of Public Health, vol. 96, no. 2, pp. 97-101, 2005.

[10] A. Sarang, T. Rhodes, L. Platt et al., "Drug injecting and syringe use in the HIV risk environment of Russian penitentiary institutions: qualitative study," Addiction, vol. 101, no. 12, pp. 1787-1796, 2006.

[11] "Women in Prison, A Review of the Conditions in Member States of the Council of Europe," The Quaker Council of European Affairs, 2007. 
[12] S. Fazel, P. Bains, and H. Doll, "Substance abuse and dependence in prisoners: a systematic review," Addiction, vol. 101, no. 2, pp. 181-191, 2006.

[13] D. Rickford, "Troubled inside: responding to the mental health needs of women in prison," Prison Reform Trust, pp. 4-17, 2003.

[14] D. C. Doll, "Tattooing in prison and HIV infection," The Lancet, vol. 1, no. 8575-8576, pp. 66-67, 1988.

[15] United Nations Office On Drugs and Crime UNODC and Crime (UNODC), "Women and HIV in prison settings," 2008, http://www.unodc.org/documents/hiv-aids/Women $\% 20$ and\%20HIV\%20in\%20prison\%20settings.pdf.

[16] United Nations Office On Drugs and Crime UNODC, "HIV/AIDS prevention and care for female injecting drug users," 2006, http://www.unodc.org/pdf/HIV-AIDS_ femaleIDUs_Aug06.pdf.

[17] N. Waring and B. Smith, "The AIDS epidemic: impact on women prisoners in Massachusetts-an assessment with recommendations," Women and Criminal Justice, vol. 2, pp. 117-143, 1991.

[18] B. H. Zaitzow, "Whose problem is it anyway? Women prisoners and HIV/AIDS," International Journal of Offender Therapy and Comparative Criminology, vol. 45, no. 6, pp. 673-690, 2001.

[19] B. H. Zaitow and A. D. West, "Doing time in the shadow of death: women prisoners and HIV/AIDS," in The Incarcerated Woman: Rehabilitative Programming in Women's Prisons, S. F. Sharp, Ed., pp. 73-90, Prentice Hall, Upper Saddle River, NJ, USA, 2003.

[20] C. A. Franklin, N. E. Fearn, and T. W. Franklin, "HIV/AIDS among female prison inmates: a public health concern," Californian Journal of Health Promotion, vol. 3, no. 2, pp. 99-112, 2005.

[21] T. Hammett, P. Harmon, and L. Maruschak, 1996-1997 Update: HIV/AIDS, STDs, and TB in Correctional Facilities, U.S. Department of Justice, National Institute of Justice, Washington, DC, USA, 1999.

[22] L. M. Maruschak, HIV in Prisons and Jails, 2002. Bureau of Justice Statistics Bulletin, U.S. Department of Justice, Office of Justice Programs, Washington, DC, USA, 2004.

[23] S. Mushayabasa, C. P. Bhunu, and R. J. Smith, "Assessing the impact of educational campaigns on controlling HCV among women in prison settings," Communications in Nonlinear Science and Numerical Simulation, vol. 17, no. 4, pp. 1714-1724, 2012.

[24] C. S. Pedamallu, L. Ozdamar, E. Kropat, and G.-W. Weber, "A system dynamics model for intentional transmission of HIV/AIDS using cross impact analysis," Central European Journal of Operations Research, vol. 20, no. 2, pp. 319-336, 2012.

[25] P. Van Den Driessche and J. Watmough, "Reproduction numbers and sub-threshold endemic equilibria for compartmental models of disease transmission," Mathematical Biosciences, vol. 180, pp. 29-48, 2002.

[26] J. Carr, Applications of the Centre Manifold Theory, Springer, New York, NY, USA, 1981.

[27] C. Castillo-Chavez and B. Song, "Dynamical models of tuberculosis and their applications," Mathematical Biosciences and Engineering, vol. 1, no. 2, pp. 361-404, 2004.

[28] D. Green and W. Al-Fwzan, "An improved optimistic threestage model for the spread of HIV amongst injecting intravenous drug users," Discrete and Continuous Dynamical Systems, pp. 286-299, 2009.
[29] C. P. Bhunu, W. Garira, and Z. Mukandavire, "Modeling HIV/AIDS and tuberculosis coinfection," Bulletin of Mathematical Biology, vol. 71, no. 7, pp. 1745-1780, 2009.

[30] C. P. Bhunu, S. Mushayabasa, and J. M. Tchuenche, "A theoretical assessment of the effects of smoking on the transmission dynamics of tuberculosis," Bulletin of Mathematical Biology, vol. 73, no. 6, pp. 1333-1357, 2011. 

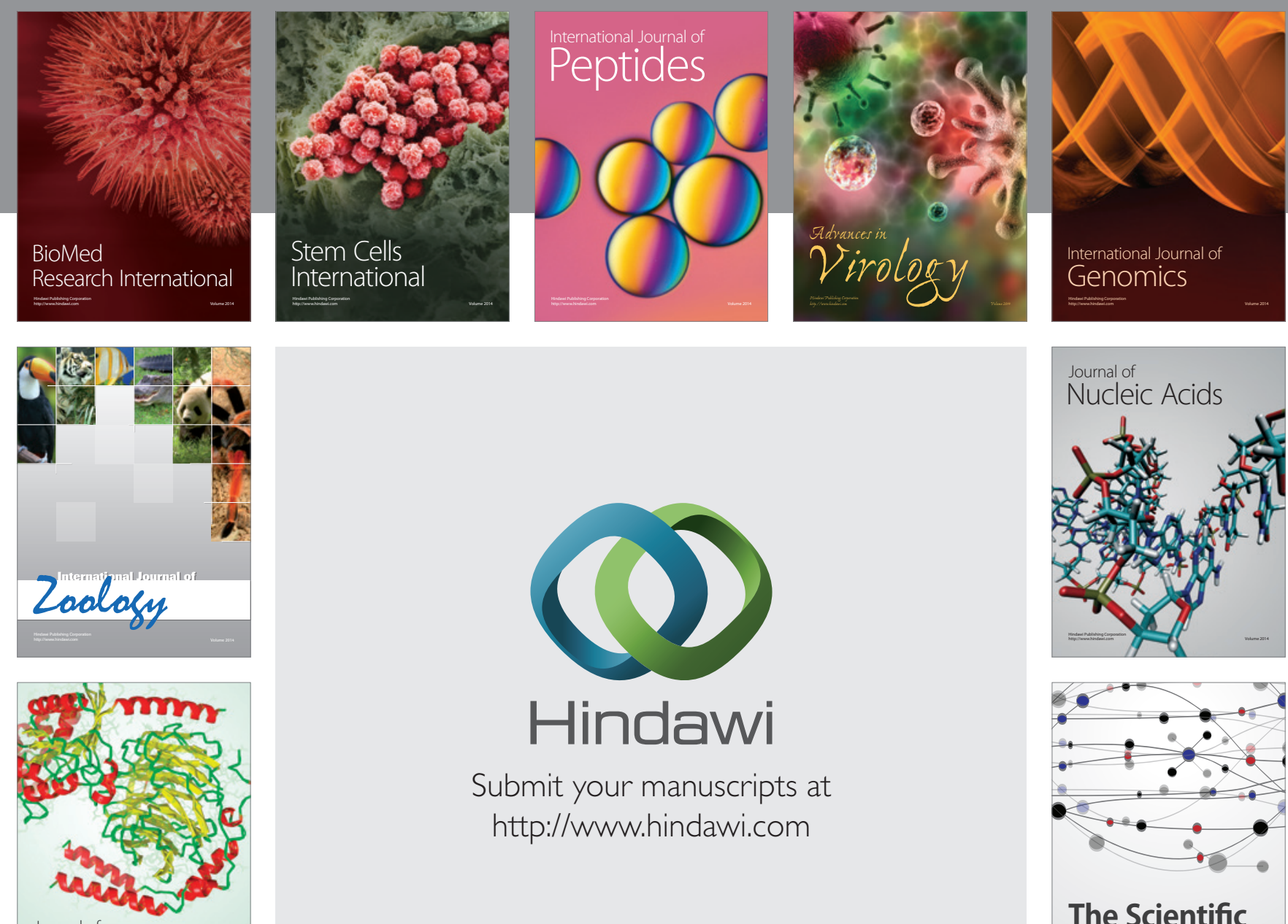

Submit your manuscripts at

http://www.hindawi.com

Journal of
Signal Transduction
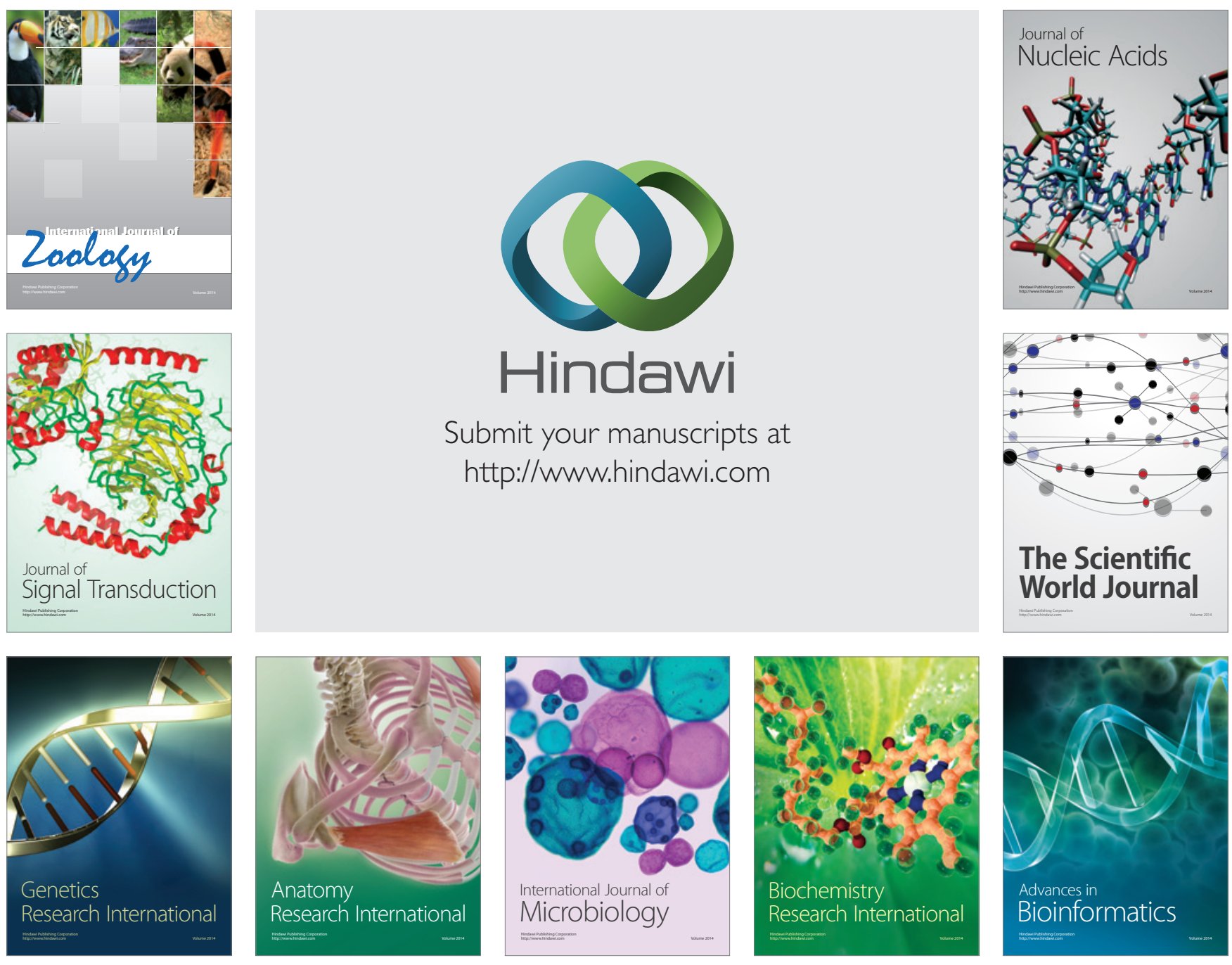

The Scientific World Journal
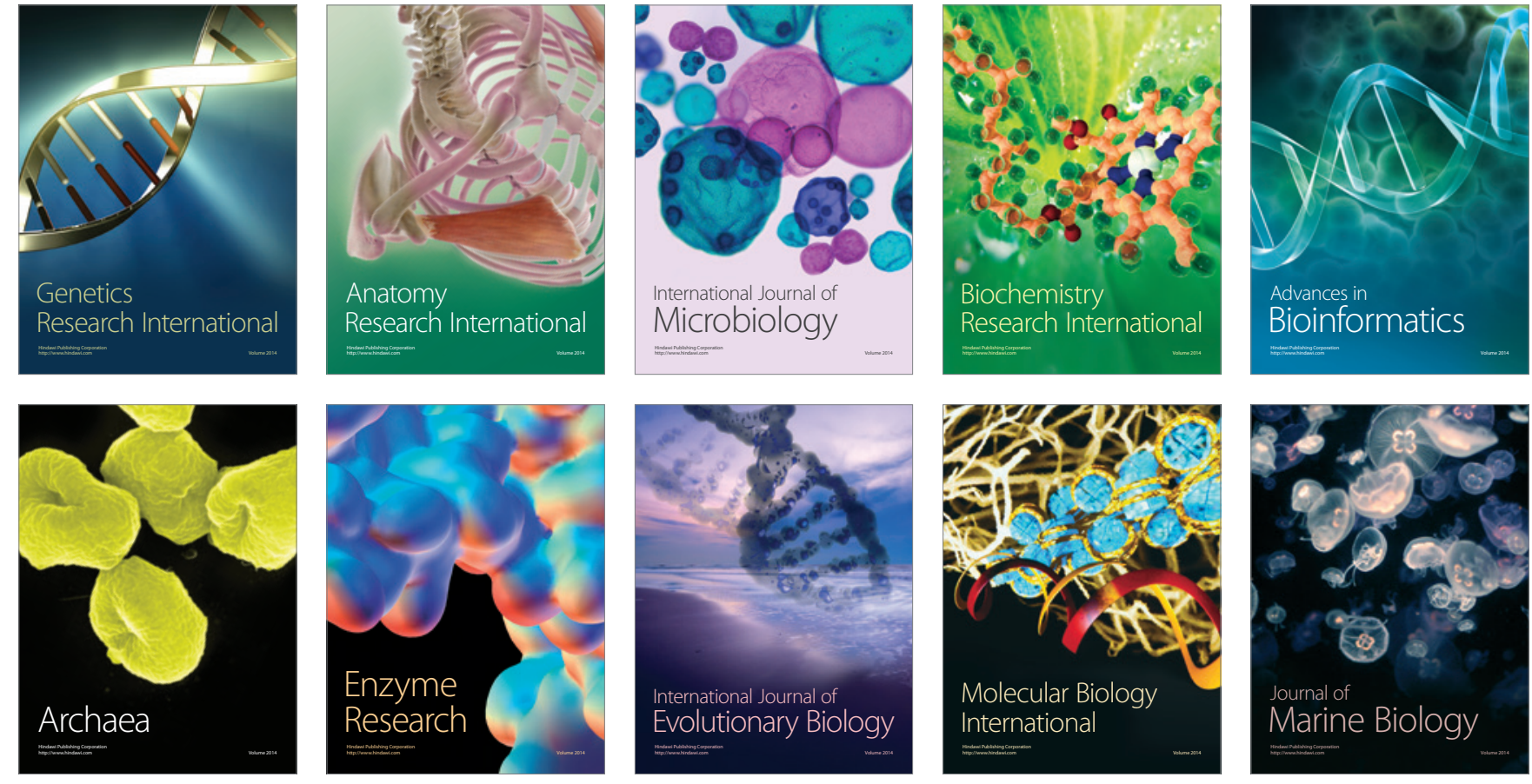